\title{
Different fusion tags affect the activity of ubiquitin overexpression on spastin protein stability
}

\author{
Jianyu Zou, ${ }^{1 *}$ Zhenbin Cai, ${ }^{1 *}$ Zhi Liang, ${ }^{2 *}$ Yaozhong Liang, ${ }^{1}$ Guowei Zhang, ${ }^{1}$ Jie Yang, ${ }^{1}$ Yunlong Zhang, \\ Hongsheng Lin, ${ }^{1}$ Minghui Tan ${ }^{1}$ \\ ${ }^{1}$ Department of Orthopaedics, The First Affiliated Hospital of Jinan University, Guangzhou \\ ${ }^{2}$ Department of Orthopaedics, The Eighth Affiliated Hospital of Sun Yat-sen University, Shenzhen, China
}

*These authors contributed equally to this work.

Spastin is one of the proteins which lead to hereditary spastic paraplegia (HSP), whose dysfunction towards microtubule severing and membrane transporting is critically important. The present study is to elucidate the mechanisms of the protein stability regulation of spastin. The ubiquitin encoding plasmids were transfected into COS-7 cells with different fusion tags including Green Fluorescent Protein (GFP), mCherry and Flag. The expression level of spastin was detected, microtubule severing activity and neurite outgrowth were quantified. The data showed that ubiquitin overexpression significantly induced the decreased expression of spastin, suppressed the activity of microtubule severing in COS-7 cells and inhibited the promoting effect on neurite outgrowth in cultured hippocampal neurons. Furthermore, when modulating the overexpression experiments of ubiquitin, it was found that relatively small tag like Flag, but not large tags such as GFP or mCherry fused with ubiquitin, retained the activity on spastin stability. The present study investigated the effects of small/large tags addition to ubiquitin and the novel mechanisms of post-transcriptional modifications of spastin on regulating neurite outgrowth, in the attempt to experimentally elucidate the mechanisms that control the level or stability of spastin in hereditary spastic paraplegia.

Key words: Spastin; ubiquitination; protein stability; microtubule severing; neurite outgrowth.

Correspondence: Dr. Minghui Tan, Department of Orthopaedics, the First Affiliated Hospital of Jinan University, Guangzhou 510630, China. E-mail: tanminghui@jnu.edu.cn

Conflict of interest: The authors declare no conflict of interest.

Ethical approval: All procedures related to animals were applied following the Guide for the Care and Use of Laboratory Animals of the National Institutes of Health and approved by the Jinan University Institutional Animal Care and Use Committee. 


\section{Introduction}

Hereditary spastic paraplegia (HSP) is a genetically heterogeneous neurodegenerative disorder, characterized by progressive spasticity and weakness of the bilateral lower extremities due to degeneration of the bilateral corticospinal tract axons. ${ }^{1}$ Mutations in the gene encoding for SPAST (SPG4) are the most common cause of autosomal dominant HSP. ${ }^{2}$ Spastin belongs to the ATPase associated with various cellular activities (AAA) protein family and its activity is associated with diverse cellular processes. SPG4 gene translates to two spastin isoforms, M1 (68 kDa) and M87 (60 $\mathrm{kDa}){ }^{3}$ These two isoforms can produce another two isoforms (M1 $\Delta$ exon4 and M87 $\Delta$ exon4) through alternative mRNA splicing of exon4. ${ }^{4}$ Mutations of spastin in HSP include nonsense, frameshift, splicing and exon deletion, which change the activity of spastin by affecting its ATP binding, ATP hydrolysis or oligomerization. ${ }^{3}$ This haploinsufficiency of mutations likely induced the loss of function of spastin. ${ }^{5}$ Considering the essential function of spastin during many processes including microtubule severing, 6,7 endosome trafficking, ${ }^{8-11}$ and neurite extension. ${ }^{12,13}$ The loss of spastin could cause axonal transport dysfunction and axonal swellings. ${ }^{14-17}$ Lopes et al. found that spastin-involved axonal transport disorder might be related to kinesin, and eventually cause the cognitive function of mice to decline. ${ }^{11}$ In zebrafish embryos, reduced spastin can cause dramatic defects in motor axon outgrowth. ${ }^{14}$ In addition, we also found that the long non-coding RNA (LncRNA) MALAT1 regulates the expression of spastin by targeting miR-30, thereby regulating its functions in microtubule severing and neurite outgrowth. ${ }^{13}$ Surprisingly, there are few studies on the degradation mechanism of spastin. Sardina et al. found that HIPK2 (Homeodomain interacting protein kinase 2) can phosphorylate spastin at S268. ${ }^{15}$ This phosphorylation may inhibit the polyubiquitination and degradation of spastin. ${ }^{16}$ However, the mechanisms of how to control the protein level or stability of spastin and its effect on microtubule severing and axon outgrowth remain to be elucidated.

Ubiquitin ( $\mathrm{Ub}$ ) is a highly conserved small molecule protein widely found in eukaryotes which consists of 76 amino acids. ${ }^{17}$ The main function of the Ub system is proteasomal degradation with a range of substrates. Ub is attached to substrates by a complicated three-step enzymatic cascade action, including Ub activation by $\mathrm{E} 1, \mathrm{Ub}$ conjugation by $\mathrm{E} 2$ and $\mathrm{Ub}$ ligation by a variety of E3 enzymes. ${ }^{18-20}$ In addition to regulating protein degradation, ubiquitination events are also involved in nuclear transport, ${ }^{21}$ mitochondrial translocation ${ }^{22}$ and transcriptional activity. ${ }^{23}$ When experimentally observing the potential proteasomal degradation of a protein, overexpression of tag-fused Ub protein is commonly used, such as Green Fluorescent Protein (GFP), mCherry or Flag tags. However, whether different types of tags would impact Ub activity, especially on spastin protein stability, remains to be elucidated.

With the development of transfection technology, recombinant protein has been widely used in many studies. ${ }^{24,25}$ Macromolecular tags such as GFP and mCherry are welcomed because of their visual characteristics. However, some scholars believe that such macromolecular tags may have an impact on protein function. ${ }^{26,27}$ Therefore, they prefer to choose small peptide tags. However, there is still no report on the influence of the selection of recombinant $\mathrm{Ub}$ fusion tag on its protein function, especially on the activity of spastin.

The present study investigated whether spastin stability could be regulated by $\mathrm{Ub}$ expression with different fusion tags and further investigated the function alteration of spastin on microtubule severing and neurite outgrowth under the treatment of Ub overexpression, in order to discover the regulation mechanism of spastin.

\section{Materials and Methods}

\section{Plasmid construction}

The Ub gene was synthesized (Invitrogen; Thermo Fisher Scientific, Inc., Waltham, MA, USA) and subcloned into vectors including pEGFP-C1, pmCherry-C1, pECMV-3X-Flag and pCMV-HA (Clontech Laboratories, Inc., Mountain View, CA, USA). The methods used for constructing cDNA plasmids was performed according to a previous study. ${ }^{28}$ All the constructs were sequenced and verified. The following primer pairs were used for PCR amplification: GFP-Ub forward, tacaagtccggactcagatctATG CAGATCTTCGTGAAAACCC and reverse, gtaccgtcgactgcaga attcACCACCTCTCAGACGCAGGA; mCherry-Ub forward, gacgagctgtacaagagatctATGCAGATCTTCGTGAAAACCC and reverse, gtaccgtcgactgcagaattcACCACCTCTCAGACGCAGGA; Flag-Ub forward, cttggtaccgagctcggatccGCCACCATGCAGA TCTTCGTGAAAACCC and reverse: aacgggecctctagactcgag ACCACCTCTCAGACGCAGGA and HA-Spastin forward, ggaggccegaattcggtcgacATGGCCGCCAAGAGGAGC and reverse, catgtctggatcccegcggecgcTTAAACAGTG GTGTCTCCAAAGTCC.

\section{Cell culture and transfection}

COS-7 (SCSP-508) cells were purchased from the Shanghai Institute of Biochemistry and Cell Biology, Chinese Academy of Sciences. COS-7 cells were cultured in Dulbecco's Modified Eagle Medium (DMEM)/F12 (Gibco; Thermo Fisher Scientific, Inc.) supplemented with 10\% fetal bovine serum (FBS) (Gibco; Thermo Fisher Scientific, Inc.) in a $37^{\circ} \mathrm{C}$ and $5 \% \mathrm{CO}_{2}$ incubator. Half of the culture media was replaced every 3 days. COS-7 cells were seeded into six-well plates (Corning). Cells were transiently transfected with GFP-ubiquitin, mCherry-ubiquitin, or Flag-ubiquitin $(4.0 \mu \mathrm{g}$ of plasmid per well). Transient transfection of plasmids was performed using Lipofectamine 2000 ${ }^{\mathrm{TM}}$ (Invitrogen; Thermo Fisher Scientific, Inc.) according to the manufacturer's instructions. After transfection, cells were grown 36-48 h before harvesting. Hippocampal neurons of newborn Sprague-Dawley rat pups were performed as previously described. ${ }^{28,29}$ Hippocampi were dissected and obtained by $0.125 \%$ trypsin (Thermo Fisher Scientific, Inc.), and then plated onto poly-D-lysine-coated glass coverslips (Thermo Fisher Scientific, Inc.), at a density of $1 \times 10^{4}$ cells $\mathrm{cm}^{2}$. Neuronal cultures were maintained in Neurobasal-A medium (Gibco; Thermo Fisher Scientific, Inc.) containing 2\% B27 (Gibco; Thermo Fisher Scientific, Inc.) in a humidified incubator. One-half of the culture media was replaced every 3 days. Plasmids were transfected at 3 days in vitro (DIV3) using the calcium phosphate method and cultured with complete media for another 2 days (DIV5). DIV5 neurons were fixed with 4\% paraformaldehyde (Sigma-Aldrich; Merck KGaA, Darmstadt, Germany) and then stained by immunocytochemistry.

\section{Cycloheximide treatment}

The effect of recombinant $\mathrm{Ub}$ with different tags on the stability of spastin is determined by cycloheximide (CHX) (cat. no. HY12320; MedChemExpress, Monmouth Junction, NJ, USA) processing. After transfection, the cells will continue to be cultured for 24-36 h before the next step. Cells were treated with CHX (100 $\mu \mathrm{g} / \mathrm{ml}$ ) for another $12 \mathrm{~h}$, then collected and lysed for Western Blotting.

\section{Immunofluorescence}

Immunofluorescence was performed using a previously described standard protocol. ${ }^{30}$ Cells were weed onto poly-Dlysine-coated coverslips, fixed in the $4 \%$ paraformaldehyde at $4{ }^{\circ} \mathrm{C}$ 
for $45 \mathrm{~min}$, permeabilized in 1\% Triton X-100 in Tris Buffered Saline (TBS) at room temperature for $15 \mathrm{~min}$, and then blocked in $5 \%$ bovine serum albumin (BSA) in TBS at room temperature for $1 \mathrm{~h}$ before the primary antibodies were applied. The following primary antibodies were used: Anti-GFP (cat. no. ab290; Abcam, Cambridge, UK), anti-mCherry (cat. no. ab125096; Abcam), antiTubulin (cat. no. ab6160; Abcam), anti-Flag (cat. no. F1804; Sigma-Aldrich, Merck KGaA), anti-HA (cat. no. SAB1306169; Sigma-Aldrich), all at a dilution of 1:1,000. Alexa Fluor 488 (cat. no. A-21206; Invitrogen; Thermo Fisher Scientific, Inc.), Alexa Fluor 555 (cat. no. A-31570; Invitrogen; Thermo Fisher Scientific, Inc.) and Alexa Fluor 647 (cat. nos. ab150115 and ab150167; Abcam) secondary antibodies were used at a dilution of 1:1,000 for probing. Subsequently, coverslips were mounted using Fluro-Gell II with DAPI (cat. no. 17985-50; Electron Microscopy Sciences, Hatfield, PA, USA) and images were captured using a Carl Zeiss LSM 780 confocal microscope (Zeiss AG, Oberkochen, Germany). We used cells incubated with no primary antibody as negative control. Images were acquired with the same optical slice thickness for every channel using a $63 \times$ oil objective and a resolution of $1024 \times 1024$ pixels. Images of COS-7 cells were input into Image $\mathrm{J}$ for processing. After the background was standardized, the fluorescence intensity of the microtubules was determined. At least 30 neurons from each experimental group were used for the qualification of neurite/dendrites/axons using Image-Pro Plus 6 software (Media Cybernetics, Silver Spring, MD, USA). All these procedures were performed in a blind manner, and examinations were performed at least three times.

\section{Western blotting}

Cells were harvested and lysed in lysis buffer (20 mM Tris , $\mathrm{pH}$ 7.5, $150 \mathrm{mM} \mathrm{NaCl1 \%}$ Triton X-100, $2.5 \mathrm{mM}$ sodium pyrophosphate, $1 \mathrm{mM} \mathrm{Na} \mathrm{VO}_{4}, 2 \mathrm{mM}$ EDTA, $1 \mathrm{mM}$ PMSF and protease inhibitors (cat. no. HY-K0010; MedChemExpress)) as previously described. ${ }^{30}$ Briefly, samples were loaded and separated by SDS-
PAGE, transferred to PVDF membranes (EMD Millipore) and blocked with 5\% skimmed milk (BD Bioscience) at room temperature for $1 \mathrm{~h}$. Subsequently, membranes were probed overnight at $4^{\circ} \mathrm{C}$ with the following primary antibodies: mouse anti-spastin (1:100, cat. no. sc-374068; Santa Cruz Biotechnology, Inc.), mouse anti-GAPDH (1:5,000, cat. no. ab8245; Abcam), rabbit anti-GFP (1:1,000, cat. no. ab290; Abcam), rabbit anti-mCherry (1:1,000, cat. no. ab125096; Abcam), mouse anti-Flag (1:1,000, cat. no. F1802; Sigma-Aldrich; Merck KGaA). HRP-conjugated secondary antibodies (cat. nos. AS038 and AS003; ABclonal Biotech Co, Ltd.) were used for protein signal detection with an enhanced chemiluminescence kit (Pierce; Thermo Fisher Scientific, Inc.).

\section{Statistical analysis}

Experiments were performed at least three times. GraphPad Prism 5 software was used to make graphs and perform statistical analyses. Statistical significance between two groups was determined using Student's $t$-test, and comparisons between more than two groups were performed using one-way ANOVA followed by Newnan-Keuls post-hoc test. The experimental data are expressed as mean \pm standard deviation (SD); $\mathrm{p}<0.05$ was considered to indicate a statistically significant difference.

\section{Results}

Protein stability of spastin is downregulated by ubiquitin overexpression

To investigate whether protein stability of spastin could be affected by ubiquitin, cultured COS-7 cells were transfected with $\mathrm{Ub}$ encoding plasmids with different fusion tags including GFP, mCherry and Flag. As shown in Figure 1 a-c, Flag-tagged Ub decreased the level of endogenous spastin in COS-7 cells, while (a)

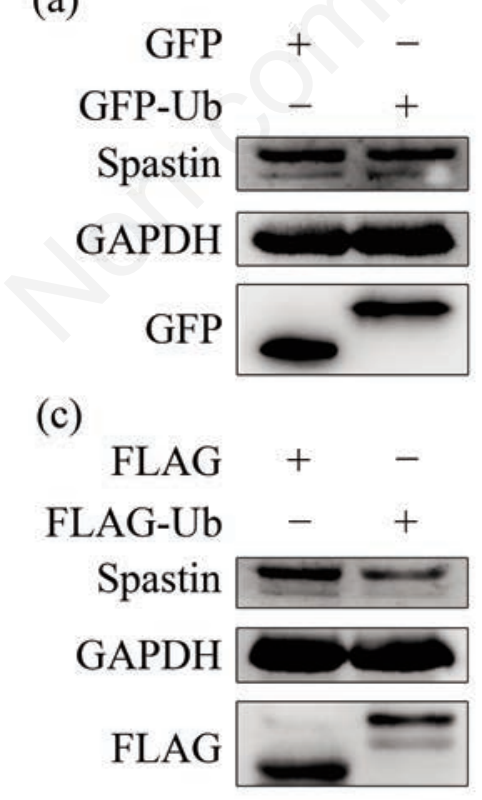

(b)

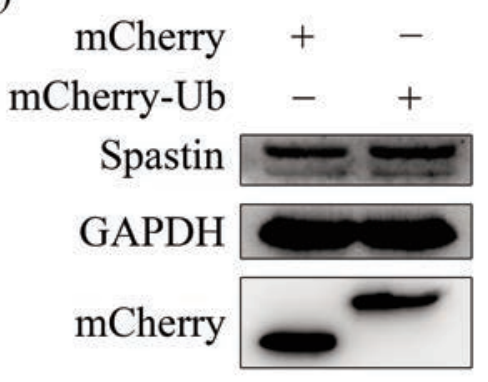

(d)

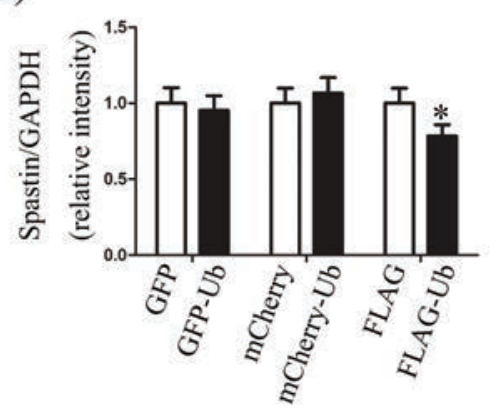

Figure 1. Protein stability of spastin is regulated by ubiquitin overexpression. a-c) Overexpression of different tagged plasmids in COS7 cells at $24 \mathrm{~h}$; cell lysates were subjected to Western blotting with antibodies against spastin, GAPDH, GFP, mCherry, and Flag. d) Quantification of band intensities of spastin relative to GAPDH. The relative value in the control group was set to 1 . Data are presented as the mean \pm standard deviation from at least three independent experiments; ${ }^{*} \mathrm{p}<0.05$ vs vector group. 
GFP- or mCherry-tagged Ub showed no effect. Western blot analysis showed that relatively short tagged $\mathrm{Ub}$ had regulation activity compared with larger ones (Figure 1d). However, direct transfection of Flag-ubiquitin encoding plasmid did not show a significant effect on spastin protein degradation, due to extensive translation (Figure 1c). Therefore, CHX was added to inhibit the transcriptional translation of spastin following transfection. As shown in Figure 2, after application of $\mathrm{CHX}$, transfection of Flag-tagged (Figure 2c) Ub almost inhibited spastin protein expression, while GFP(Figure 2a) or mCherry-tagged (Figure 2b) Ub showed no changes. The quantified data were shown in Figure 2d. These data suggested that protein stability can be regulated by Ub and short epitope tags hardly impacted the activity of $\mathrm{Ub}$ in overexpression experiments.

\section{Ubiquitin overexpression reduces the microtubule sev-} ering activity of spastin

The encoding plasmids with Flag-tag maintained Ub activity and reduced the expression of endogenous spastin in COS-7 cells, it was determined whether this lowered expression would affect the severing activity of spastin toward microtubules. Plasmids were co-transfected in COS-7 cells and microtubule cytoskeletons were stained with the indicated antibodies by immunocytochemistry. As shown in Figure 3a, overexpression of HA-spastin significantly severed microtubules, induced fragmentation structures and lowered microtubule numbers. Since GFP is used for indication of transfection, only mCherry-Ub was tested for the following experiments. As shown Figure 3b, co-expression of mCherry-Ub did not affect the expression of HA-spastin and microtubule severing activity. However, co-expression of Flag-Ub markedly reduced the immuno-stained signal of HA-spastin and rescued the severed microtubule (Figure $4 \mathrm{a}, \mathrm{b}$ ). These data indicated that Ub overexpression reduced spastin protein stability and decreased microtubule severing activity.

\section{Ubiquitin overexpression inhibits neurite outgrowth induced by spastin in cultured hippocampal neurons}

Spastin activity is critical for regulating neurite outgrowth in hippocampus neurons. ${ }^{29}$ We have previously reported that overexpression of spastin promoted neurite outgrowth in hippocampal neurons cultured in vitro. ${ }^{31}$ The present study determined whether overexpression of $\mathrm{Ub}$ in the hippocampus in vitro could regulate neurite outgrowth via modulating the stability of spastin. As shown in Figure 5, overexpression of spastin promoted the growth of neurite, as previously reported. ${ }^{31}$ Representative images of neuronal are shown in Figure $5 \mathrm{~b}$ and Sholl analysis of dendrite crossings is shown in Figure 5c. The overexpression of mCherry-Ub did not impact the effect on spastin-induced neurite outgrowth (Figure 5b). In the meantime, co-overexpression of Flag-Ub suppressed the promoting effect of spastin, as Flag-Ub overexpression reduced the length Figure $6 a, b)$ and complexity (Figure $6 c$ ) of neurons. These data suggested that spastin-promoted neurite outgrowth could be inhibited via $\mathrm{Ub}$ overexpression.

\section{Discussion}

Ubiquitin plays a very important role in the process of protein degradation in cells. The present study demonstrated that overexpression of $\mathrm{Ub}$ regulated the protein stability of spastin, decreased the microtubule severing activity of spastin and restrained its neurite outgrowth promoting effect in cultured hippocampal neurons. Furthermore, it was found that $\mathrm{Ub}$ with the small peptide tag Flag, but not GFP- or mCherry-tagged Ub with large molecule weight, retained the degradation activity towards spastin. These data suggested that spastin may be regulated via the Ub proteasome system and when modulating Ub function, especially applied in overexpression experiments, small or short epitope tags should be selected. (a)

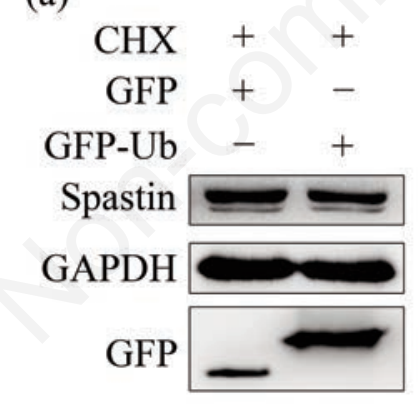

(c)

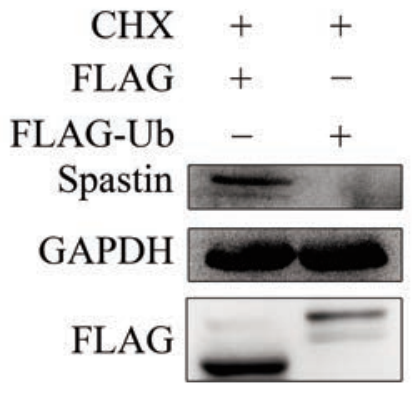

(b)

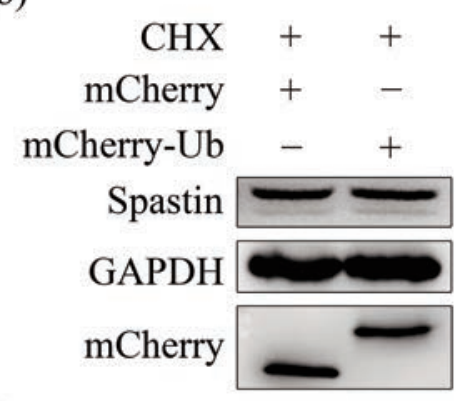

(d)

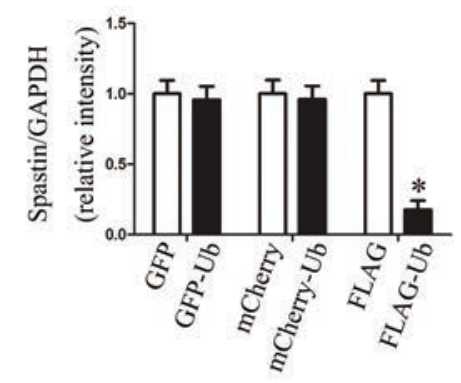

Figure 2. Flag-tagged ubiquitin affects spastin protein stability. a-c) Overexpression of different tagged plasmids in COS-7 cells, which were subsequently treated with Cycloheximide for $12 \mathrm{~h}$; cell lysates were collected and subjected to western blotting with the indicated antibodies. d) Quantification of band intensities of spastin relative to GAPDH. The relative value in the control group was set to 1. Data are presented as the mean \pm standard deviation from at least three independent experiments; ${ }^{*} p<0.05 v s$ vector group. 
Spastin, a microtubule-severing protein, promotes the growth of hippocampal neurons by cutting long microtubules to short segments. More research has focused on the role of spastin in transporting and severing, via the interaction of other proteins, includ- ing endosomal sorting complexes required for transport-III,, 32 vesicle-associated membrane protein $7,{ }^{33}$ atlastin $^{34}$ and CRMP5. ${ }^{31}$ We have previously discovered that LncRNA MALAT1 regulates the protein level of spastin via the transcriptional control. ${ }^{13}$ (a)

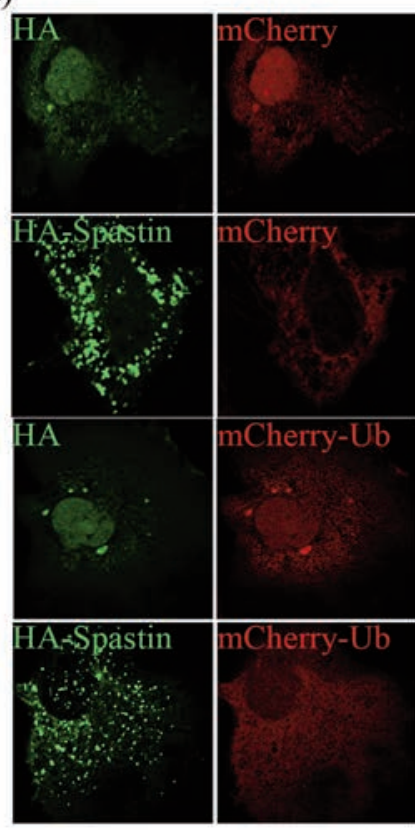

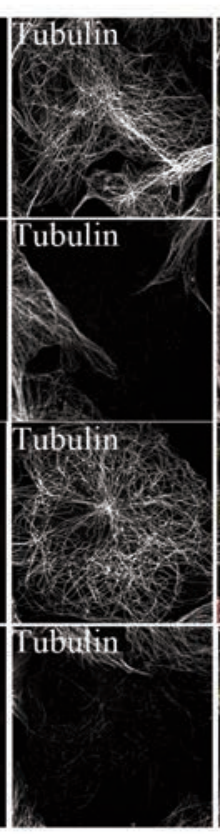

(b)

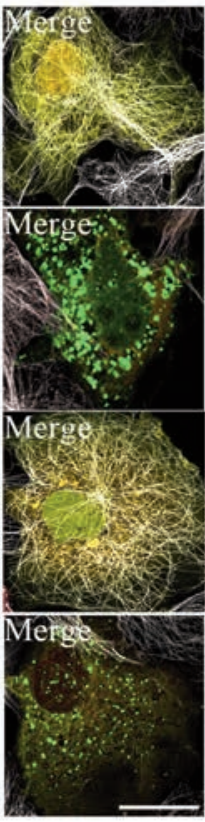

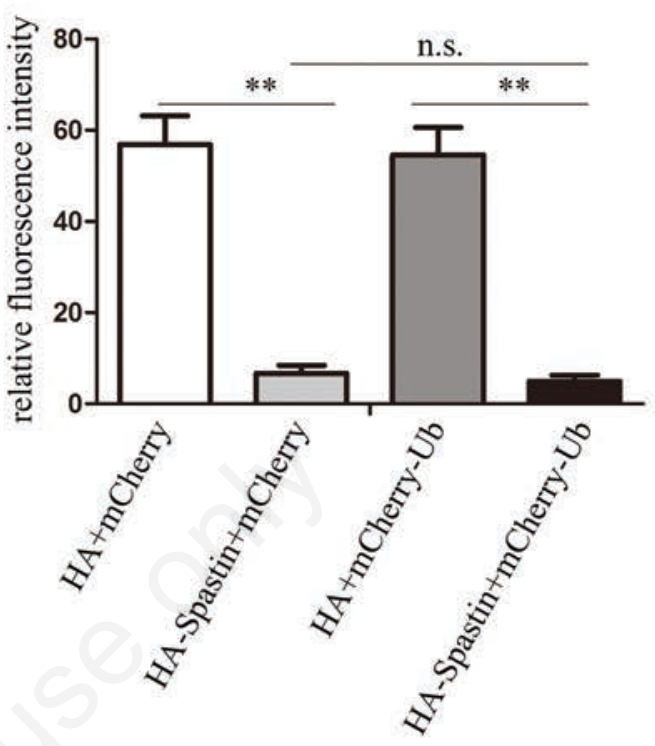

Figure 3. mCherry-tagged ubiquitin does not affect the microtubule severing activity of spastin. a) Different plasmids were co-transfected in COS-7 cells for $24 \mathrm{~h}$ and then stained by immunocytochemistry with HA (green), mCherry (red) and tubulin (white) antibodies. b) Relative fluorescence intensity of tubulin in COS-7 cells. Data are presented as the mean \pm standard deviation; $n=20$ cells from three independent experiments; ${ }^{* *}$ p $<0.01$.; n.s., no significance. Scale bar: $25 \mu \mathrm{m}$.

(a)
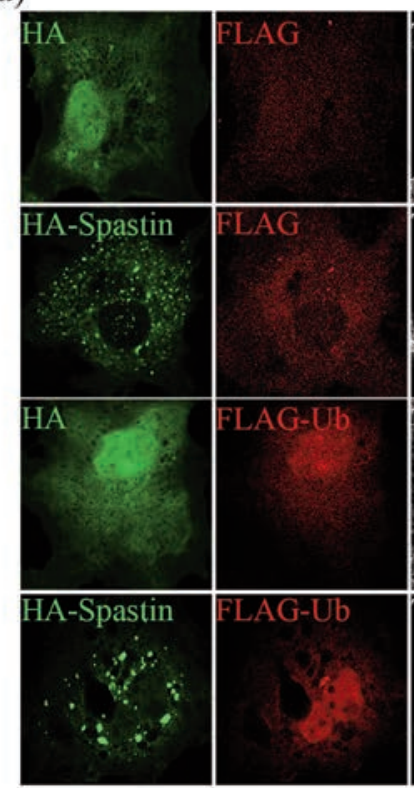
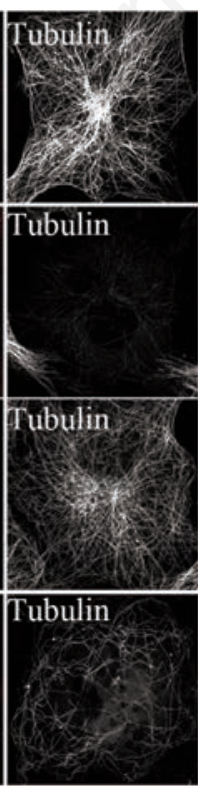

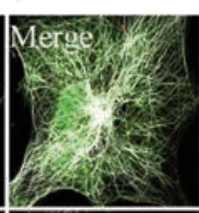

Merge

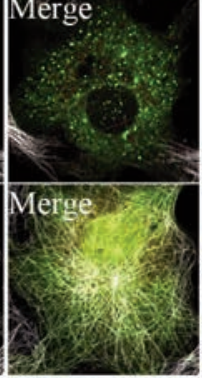

Merge

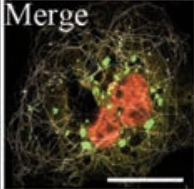

(b)

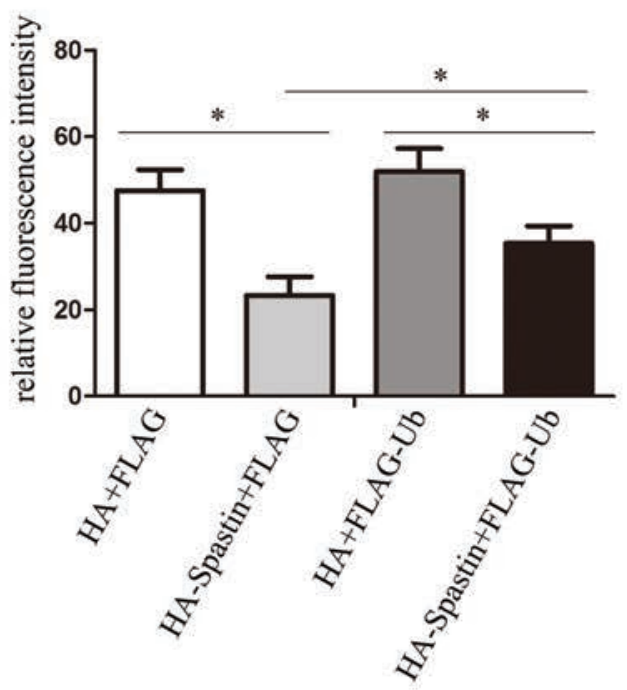

Figure 4. Flag-tagged ubiquitin suppresses the microtubule severing activity of spastin. a) Different plasmids were co-transfected as shown in Figure 3, and then stained by immunocytochemistry with HA (green), Flag (red) and tubulin (white) antibodies. b) Relative fluorescence intensity of tubulin in COS-7 cells. Data are presented as the mean \pm standard deviation; $n=20$ cells from three independent experiments; ${ }^{*} \mathrm{p}<0.05$. Scale bar: $25 \mu \mathrm{m}$. 
However, to the best of our knowledge, the post-transcriptional modification and regulation activity of spastin has rarely been reported. Recent research revealed the discovery of phosphorylation modification at S268 of spastin by HIPK2 during cell abscis- sion. ${ }^{15}$ Phosphorylation at this site will inhibit the K48-poly-ubiquitination at spastin $\mathrm{K} 544$, thereby preventing its neddylationdependent proteasomal degradation. ${ }^{16}$ The present data further confirmed that overexpression of $\mathrm{Ub}$ induced spastin instability, (a)

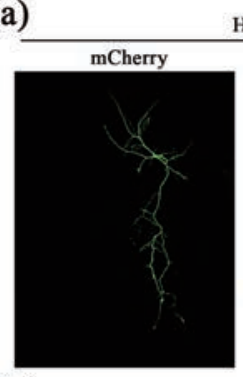

(b)

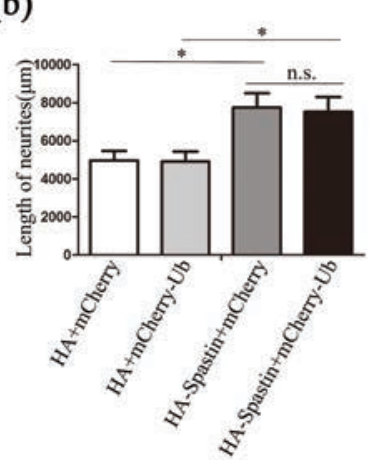

HA

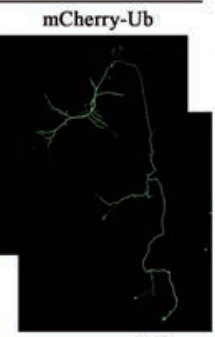

(c)
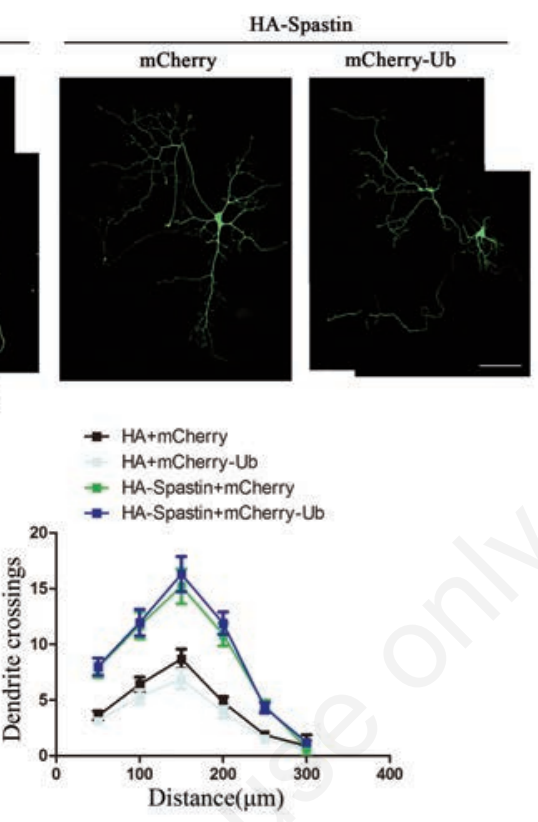

Figure 5. mCherry-tagged ubiquitin does not affect the neurite outgrowth promoting effect of spastin. a) Different plasmids with GFP vector were co-transfected in DIV3 hippocampal neurons for $48 \mathrm{~h}$ and stained by immunocytochemistry with GFP antibody to reveal the morphology; scale bar: $50 \mu \mathrm{m}$. b) The length of neuritis, including axons and dendrites. c) Sholl analysis of neurites. Data are presented as the mean \pm standard deviation; $n=20$ cells from three independent experiments; ${ }^{*} \mathbf{p}<0.05$; n.s., no significance. (a)

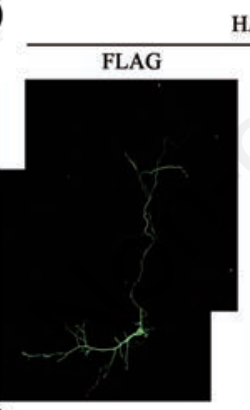

(b)

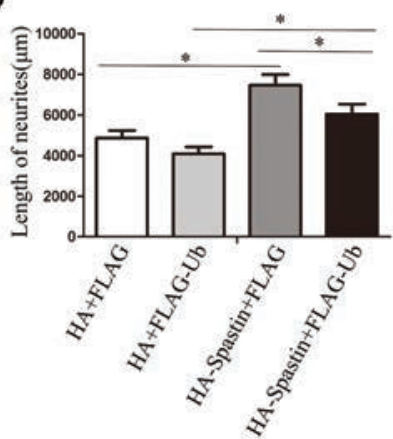

HA

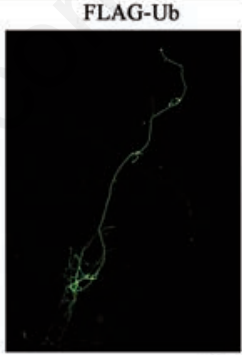

(c)
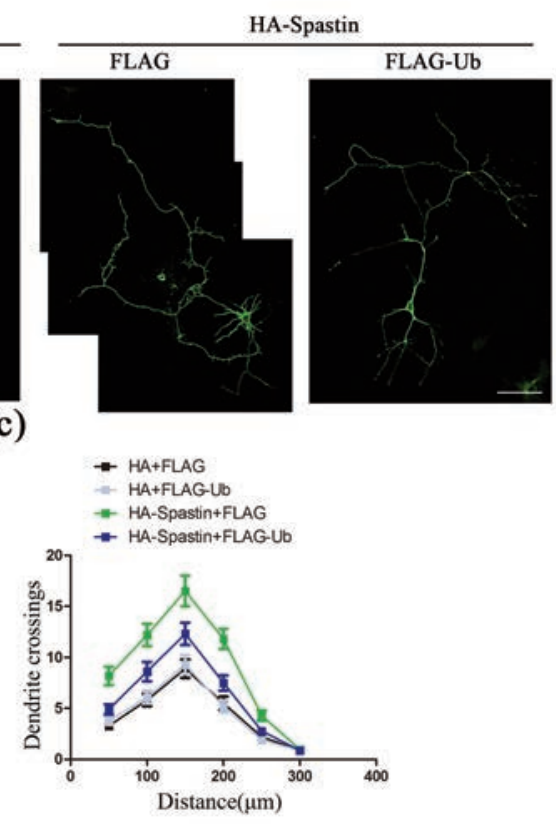

Figure 6. Flag tagged ubiquitin inhibits neurite outgrowth of spastin. a) Different plasmids were co-transfected as shown in Figure 5 and stained by immunocytochemistry to study neuronal morphology; scale bar: $50 \mu \mathrm{m}$. b) The length of neuritis, including axons and dendrites. c) Sholl analysis of neurites. Data are presented as the mean \pm standard deviation; $n=20$ cells from three independent experiments; ${ }^{*} \mathbf{p}<0.05$. 
suggesting the potential ubiquitination mechanism of spastin. However, the details of ubiquitination modification of spastin should be further clarified, which is currently under investigation in our group.

In general, ubiquitination is the most relevant PTM employed by the ubiquitin-proteasome system (UPS) and the autophagylysosome (AL) systems to label their substrates. And the strongest commonality between the two degradative systems is the small globular protein ubiquitin. ${ }^{35}$ Hence, functional Ub protein fused with different labels provides an excellent approach in the study of the Ub proteasome system. Ub is a small molecule protein, and it is unclear whether the constructed plasmid will affect the spatial structure and biological activity. The expression and purification of recombinant proteins have become increasingly common for characterizing the structure and function of proteins in recent years. However, in the selection of recombinant protein tags, different tags have their own advantages and disadvantages. The present study constructed $\mathrm{Ub}$ vectors with relatively large tags, including GFP and mCherry, and the short tag FLAG to compare the activity of Ub on spastin stability. Our data found that only small tags such as Flag, rather than larger protein tags such as GFP and mCherry, can retain the protein activity of ubiquitin. One of the concerns that people have about recombinant proteins is that the potential functional perturbance on a protein. Some studies reported the effects induced by tags, including inhibition of interactions, aberrant oligomerization and mislocalization of the recombinant protein. ${ }^{36-}$ ${ }^{38}$ It is thought that large tags enhanced the soluble expression of recombinant proteins, they can sometimes cause misfolded and nonfunctional target proteins. ${ }^{39}$ Proper protein folding plays an important role in the expression of cloned genes. ${ }^{40}$ Furthermore, contrary to large tags such as GFP and mCherry, the small-size tags have the benefits of minimizing the effect on the structure, activity, and characteristics of the recombinant protein. ${ }^{26}$ Hence, the size of a tag might be responsible for perturbing the physiological protein function. ${ }^{27}$ In order to reduce the influence of tags on the function and subcellular localization of the target protein, many researchers prefer to choose small tags (e.g., FLAG, Myc, and HA). ${ }^{26}$ The FLAG-tag system utilizes a short, hydrophilic 8-amino-acid peptide (DYKDDDDK) that is fused to the protein of interest, which is widely used in experiments of various cell types. Although the exact mechanisms were not determined, we hypothesize that large and long tags, compared with 76-AA Ub protein, may inhibit Ub transfer between the E1, E2 or E3 enzymes, or to the targeted substrate, which warrants further studies.

The present study showed that overexpression of $\mathrm{Ub}$ affected the protein stability of spastin, decreased its microtubule severing activity and restrained its neurite outgrowth promoting effect of spastin in cultured hippocampal neurons. GFP- or mCherry-tagged $\mathrm{Ub}$ showed no functional activity, while Flag-tagged Ub maintained the degradation activity towards spastin.

\section{Acknowledgements}

This work was supported by the National Natural Science Foundation of China (nos. 32071033, 81771331, and 32170977).

\section{References}

1. Blackstone C. Hereditary spastic paraplegia. Handb Clin Neurol 2018;148:633-52.

2. Zhu Z, Zhang C, Zhao G, Liu Q, Zhong P, Zhang M, et al. Novel mutations in the SPAST gene cause hereditary spastic paraplegia. Parkinsonism Relat Disord 2019;69:125-33.

3. Solowska JM, Baas PW. Hereditary spastic paraplegia SPG4: what is known and not known about the disease. Brain 2015;138:2471-84.

4. Claudiani P, Riano E, Errico A, Andolfi G, Rugarli EI. Spastin subcellular localization is regulated through usage of different translation start sites and active export from the nucleus. Exp Cell Res 2005;309:358-69.

5. Roll-Mecak A, Vale RD. Structural basis of microtubule severing by the hereditary spastic paraplegia protein spastin. Nature 2008;451:363-7.

6. Solowska JM, D'Rozario M, Jean DC, Davidson MW, Marenda DR, Baas PW. Pathogenic mutation of spastin has gain-of-function effects on microtubule dynamics. J Neurosci 2014;34:1856-67.

7. Kuo YW, Trottier O, Mahamdeh M, Howard J. Spastin is a dual-function enzyme that severs microtubules and promotes their regrowth to increase the number and mass of microtubules. Proc Natl Acad Sci USA 2019;116:5533-41.

8. Allison R, Lumb JH, Fassier C, Connell JW, Ten Martin D, Seaman MN, et al. An ESCRT-spastin interaction promotes fission of recycling tubules from the endosome. J Cell Biol 2013;202:527-43.

9. Connell JW, Allison RJ, Rodger CE, Pearson G, Zlamalova E, Reid E. ESCRT-III-associated proteins and spastin inhibit protrudin-dependent polarised membrane traffic. Cell Mol Life Sci 2020;77:2641-58.

10. Chang CL, Weigel AV, Ioannou MS, Pasolli HA, Xu CS, Peale $\mathrm{DR}$, et al. Spastin tethers lipid droplets to peroxisomes and directs fatty acid trafficking through ESCRT-III. J Cell Biol 2019;218:2583-99.

11. Lopes AT, Hausrat TJ, Heisler FF, Gromova KV, Lombino FL, Fischer T, et al. Spastin depletion increases tubulin polyglutamylation and impairs kinesin-mediated neuronal transport, leading to working and associative memory deficits. PLoS Biol 2020;18:e3000820.

12. Zhang C, Li D, Ma Y, Yan J, Yang B, Li P, et al. Role of spastin and protrudin in neurite outgrowth. J Cell Biochem 2012;113:2296-307.

13. Jiang T, Cai Z, Ji Z, Zou J, Liang Z, Zhang G, et al. The lncRNA MALAT1/miR-30/spastin axis regulates hippocampal neurite outgrowth. Front Cell Neurosci 2020;14:555747.

14. Wood JD, Landers JA, Bingley M, McDermott CJ, ThomasMcArthur V, Gleadall LJ, et al. The microtubule-severing protein Spastin is essential for axon outgrowth in the zebrafish embryo. Hum Mol Genet 2006;15:2763-71.

15. Pisciottani A, Biancolillo L, Ferrara M, Valente D, Sardina F, Monteonofrio L, et al. HIPK2 phosphorylates the microtubulesevering enzyme spastin at S268 for abscission. Cells 2019;8:684.

16. Sardina F, Pisciottani A, Ferrara M, Valente D, Casella M, Crescenzi M, et al. Spastin recovery in hereditary spastic paraplegia by preventing neddylation-dependent degradation. Life Sci Alliance 2020;3:e202000799.

17. Swatek KN, Komander D. Ubiquitin modifications. Cell Res 2016;26:399-422.

18. Deshaies RJ, Joazeiro CA. RING domain E3 ubiquitin ligases. Annu Rev Biochem 2009;78:399-434.

19. Schulman BA, Harper JW. Ubiquitin-like protein activation by E1 enzymes: the apex for downstream signalling pathways. Nat Rev Mol Cell Biol 2009;10:319-31.

20. Ye Y, Rape M. Building ubiquitin chains: E2 enzymes at work. Nat Rev Mol Cell Biol 2009;10:755-64.

21. Wang HL, He CY, Chou AH, Yeh TH, Chen YL, Li AH. Polyglutamine-expanded ataxin-7 decreases nuclear transloca- 
tion of NF-kappaB p65 and impairs NF-kappaB activity by inhibiting proteasome activity of cerebellar neurons. Cell Signal 2007; 19:573-81.

22. Bragoszewski P, Turek M, Chacinska A. Control of mitochondrial biogenesis and function by the ubiquitin - proteasome system. Open Biology 2017;7:13.

23. Geng F, Wenzel S, Tansey WP. Ubiquitin and proteasomes in transcription. Annu Rev Biochem 2012;81:177-201.

24. Kim TK, Eberwine JH. Mammalian cell transfection: the present and the future. Anal Bioanal Chem 2010;397:3173-8.

25. Vandemoortele G, Eyckerman S, Gevaert K. Pick a tag and explore the functions of your pet protein. Trends Biotechnol 2019;37:1078-90.

26. Zhao X, Li G, Liang S. Several affinity tags commonly used in chromatographic purification. J Anal Methods Chem 2013;2013:581093.

27. Hoffmann C, Gaietta G, Bünemann M, Adams SR, OberdorffMaass S, Behr B, et al. A FlAsH-based FRET approach to determine $\mathrm{G}$ protein-coupled receptor activation in living cells. Nat Methods 2005;2:171-6.

28. Zhang JF, Yin YC, Ji ZS, Cai ZB, Zhao B, Li J, et al. Endophilin2 interacts with GluA1 to mediate AMPA receptor endocytosis induced by oligomeric amyloid-beta. Neural Plasticity 2017;2017:8197085.

29. Ji ZS, Zhang GW, Chen L, Li J, Yang YH, Cha CH, et al. Spastin interacts with CRMP5 to promote neurite outgrowth by controlling the microtubule dynamics. Dev Neurobiol 2018;78:1191-205.

30. Cai ZB, Zhu XN, Zhang GW, Wu FM, Lin HS, Tan MH. Ammonia induces calpain-dependent cleavage of CRMP-2 during neurite degeneration in primary cultured neurons. Aging (Albany NY) 2019;11:4354-66.

31. Ji Z, Zhang G, Chen L, Li J, Yang Y, Cha C, et al. Spastin interacts with CRMP5 to promote neurite outgrowth by controlling the microtubule dynamics. Dev Neurobiol 2018;78:1191-205.

32. Chang CL, Weigel AV, Ioannou MS, Pasolli HA, Xu CS, Peale DR, et al. Spastin tethers lipid droplets to peroxisomes and directs fatty acid trafficking through ESCRT-III. J Cell Biol 2019;218:2583-99.

33. Plaud C, Joshi V, Marinello M, Pastre D, Galli T, Curmi PA, et al. Spastin regulates VAMP7-containing vesicles trafficking in cortical neurons. Biochim Biophys Acta Mol Basis Dis 2017; 1863:1666-77.

34. Park SH, Zhu PP, Parker RL, Blackstone C. Hereditary spastic paraplegia proteins REEP1, spastin, and atlastin-1 coordinate microtubule interactions with the tubular ER network. J Clin Invest 2010;120:1097-110.

35. Grumati P, Dikic I. Ubiquitin signaling and autophagy. J Biol Chem 2018;293:5404-13.

36. Landgraf D, Okumus B, Chien P, Baker TA, Paulsson J. Segregation of molecules at cell division reveals native protein localization. Nat Methods 2012;9:480-2.

37. Majorek KA, Kuhn ML, Chruszcz M, Anderson WF, Minor W. Double trouble-buffer selection and His-tag presence may be responsible for nonreproducibility of biomedical experiments. Protein Sci 2014;23:1359-68.

38. Song J, Markley JL. Cautionary tail: the presence of an N-terminal tag on dynein light-chain Roadblock/LC7 affects its interaction with a functional partner. Protein Pept Lett 2007; 14:265-8.

39. Biancucci M, Dolores JS, Wong J, Grimshaw S, Anderson WF, Satchell KJF, et al. New ligation independent cloning vectors for expression of recombinant proteins with a self-cleaving CPD/6xHis-tag. BMC Biotechnol 2017;17:1.

40. Einhauer A, Schuster M, Wasserbauer E, Jungbauer A. Expression and purification of homogenous proteins in Saccharomyces cerevisiae based on ubiquitin-FLAG fusion. Protein Expr Purif 2002;24:497-504.

Received for publication: 25 October 2021. Accepted for publication: 23 November 2021.

This work is licensed under a Creative Commons Attribution-NonCommercial 4.0 International License (CC BY-NC 4.0).

(C) Copyright: the Author(s), 2021

Licensee PAGEPress, Italy

European Journal of Histochemistry 2021; 65:3352

doi:10.4081/ejh.2021.3352 